\title{
Recurrent Massive Malignant Fibrous Histiocytoma of Maxilla-A Rare Case
}

\author{
1Jaimanti Bakshi, ${ }^{2}$ Roshan K Verma, 35anjeev Bhagat \\ ${ }^{1,2}$ Assistant Professor, Department of Otolaryngology, Head and Neck Surgery, Postgraduate Institute of Medical Education and \\ Research, Chandigarh, India \\ ${ }^{3}$ Senior Resident, Department of Otolaryngology, Head and Neck Surgery, Postgraduate Institute of Medical Education and \\ Research, Chandigarh, India
}

Correspondence: Jaimanti Bakshi, Assistant Professor, Department of Otolaryngology, Head and Neck Surgery, Postgraduate Institute of Medical Education and Research, Chandigarh-160012, India, Phone: 91-9914209764, Fax: +91-172-2744401 e-mail: drjayabakshi@ymail.com, roshanverma@hotmail.com

\begin{abstract}
We present a case of recurrent extensive malignant fibrous histiocytoma of maxilla. The case is unusual because of its massive extent at presentation even after primary surgery and full course of radiotherapy and chemotherapy. Even such massive tumor can be excised successfully. Primary malignant histiocytoma is rare; and such huge tumor at presentation is also rare and favorable outcome even rarer.

Keywords: Recurrent, massive, malignant fibrous histiocytoma of maxilla.
\end{abstract}

\section{INTRODUCTION}

Malignant fibrous histiocytoma (MFH) is a highly malignant primary neoplasm of the bone. The most common sites of occurrence in the head and neck are the sinonasal tract, soft tissues of the neck, craniofacial bones, and salivary glands. ${ }^{1}$ Primary malignant fibrous histiocytoma of maxilla is very rare. A literature review showed around 62 welldocumented cases of malignant fibrous histiocytoma arising at maxilla, maxillary sinuses and zygoma ${ }^{2}$ but none such extensive as this case.

We report an unusual case of recurrent malignant fibrous histiocytoma of maxilla, who presented with extensive local recurrence after 8 months of surgical excision and postoperative chemoradiotherapy. At presentation, large exophytic growth was seen involving bilateral nasal cavities extending to left maxillary sinus, orbit, oral cavity and left infratemporal fossa. Successful surgery was done along with postoperative chemotherapy.

\section{CASE REPORT}

A 40 years old male patient was referred to the department of ENT and head and neck surgery to our institute, with recurrent episodes of bleeding from left nasal cavity for 3 years. The nasal bleed was spontaneous, episodic, 2-3 episodes per month. During each episode nasal bleed was profuse and used to stop spontaneously. He also had history of bilateral nasal blockade for 2 years. He also complained of mass in the oral cavity for 2 months. He was operated thrice at private hospital within 6 months for suspected nasal polyposis, twice endoscopically and patient had lost all clinical records of first two surgeries. Third time he was again operated for recurrent lesion via both endoscopic and external approach in private hospital. The tumor was involving bilateral nasal cavity maxilla, anterior and posterior ethmoids, sphenoids. There was no intracranial extension. Histopathology of excised mass was reported as malignant fibrous histiocytoma. Patient was given full course of radiotherapy and 5 cycles of chemotherapy containing cisplatin. Patient remained asymptomatic for two months. He developed similar complaints again for which he was referred to our hospital. Examination revealed blood clots and crusts on right nasal cavity. There was a $8 \times 6 \mathrm{~cm}$ swelling involving right cheek extending up to zygoma superiorly and inferiorly upto base of mandible. Oral examination revealed soft friable fleshy mass, bleeding on touch extending up to soft palate, hard palate, upper alveolus crossing midline and involving $1 / 3$ of right $1 / 2$ of upper alveolus. Patient was a known case of type 2 diabetes mellitus and was on oral hypoglycemics.

CT scan showed large lobulated heterogeneously enhancing soft tissue mass $(8 \times 5 \times 8 \mathrm{~cm})$ in the left sinonasal cavity with epicenter in maxilla causing complete obliteration of the nasal cavity and destruction of the adjoining bony 
structures with extensive locoregional infilteration. The tumor was involving whole left maxilla, nasal septum, ethmoids, sphenoids, left orbit, hard palate and adjacent zygomatic bone. Superiorly it was reaching uptil the fovea ethmoidalis. The tumor was extending laterally into infratemporal fossa. No obvious intracranial extension noted. The posterior nasopharyngeal wall was involved. MRI showed homogenous soft tissue involving left orbit, bilateral nasal cavity, maxillary sinus, bilateral ethmoid sinus extending into the subcutaneous tissue on left side of face (Fig. 1). It was hypointense on $\mathrm{T} 1$ and hyperintense on $\mathrm{T} 2$ and showed moderate contrast enhancement. It was expanding and destroying left nasal cavity, maxillary antrum and extending into left infratemporal fossa, malar, subcutaneous tissue on left side. Inferiorly the tumor was destroying extending into oral cavity with destruction of hard palate. Superiorly, was tumor involving left orbit with destruction of medial and inferior wall with ill-defined fat planes. Chest X-ray and abdominal ulatrasound showed no evidence of metastasis. Routine blood investigations were normal.

The patient underwent left radical maxillectomy with orbital exenteration by modified Weber-Ferguson approach (Fig. 2). During the procedure small CSF leak occured from fovea ethmoidalis which was repaired by fat and surgical. Medicated pack was put in the cavity which was removed on 5th day.

Histopathological examination of excised specimen showed tumor arranged predominantly in form of fascicular pattern and storiform pattern with areas of hemorrhage (Figs 3 and 4). Background showed areas of myxoid

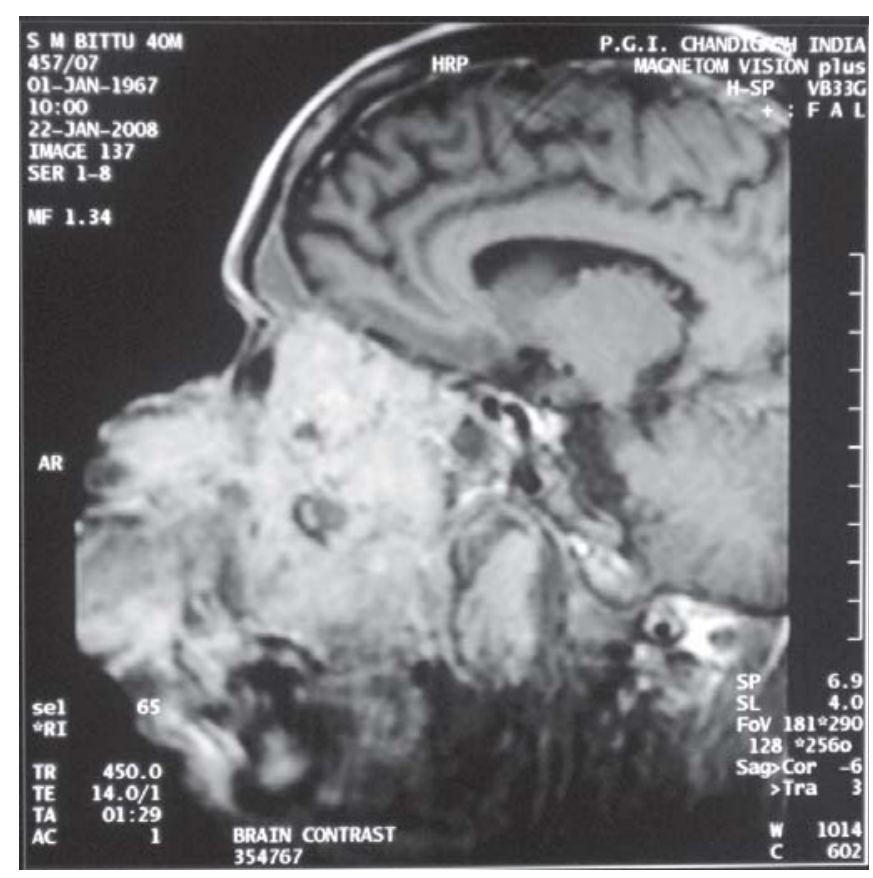

Figure 1: MRI-sagittal cut showing the extent of lesion

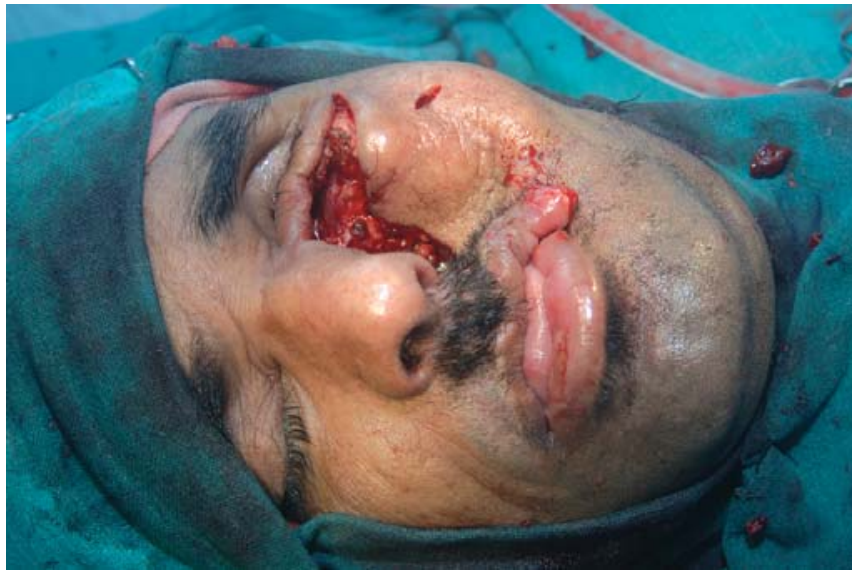

Figure 2: Intraoperative and postoperative photograph of patient showing Weber-Fergusson approach to excise the lesion

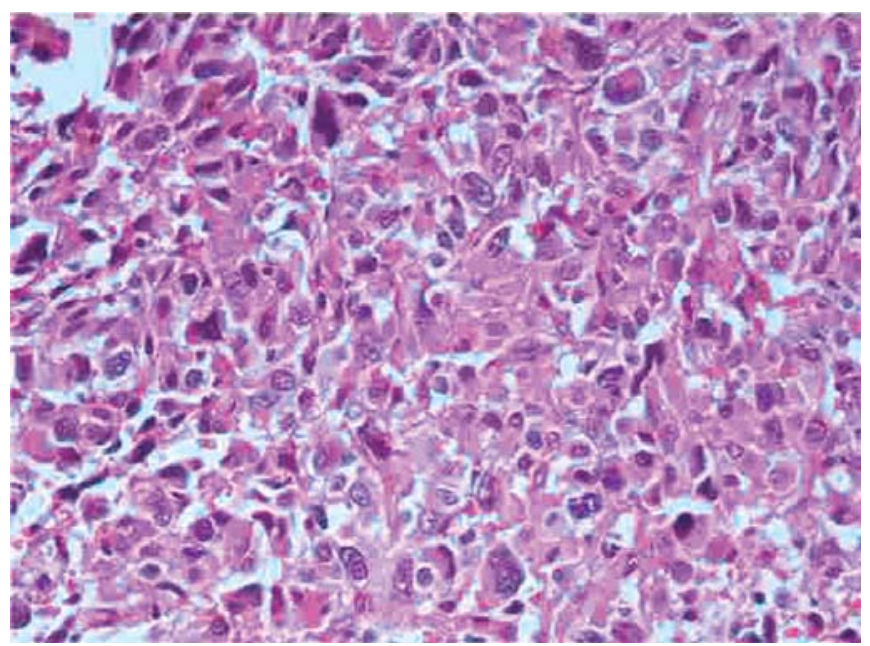

Figure 3: Photomicrograph of malignant fibrous histiocytoma showing fascicular pattern of pleomorphic spindle shaped tumor cells (hematoxylin and eosin, X100)

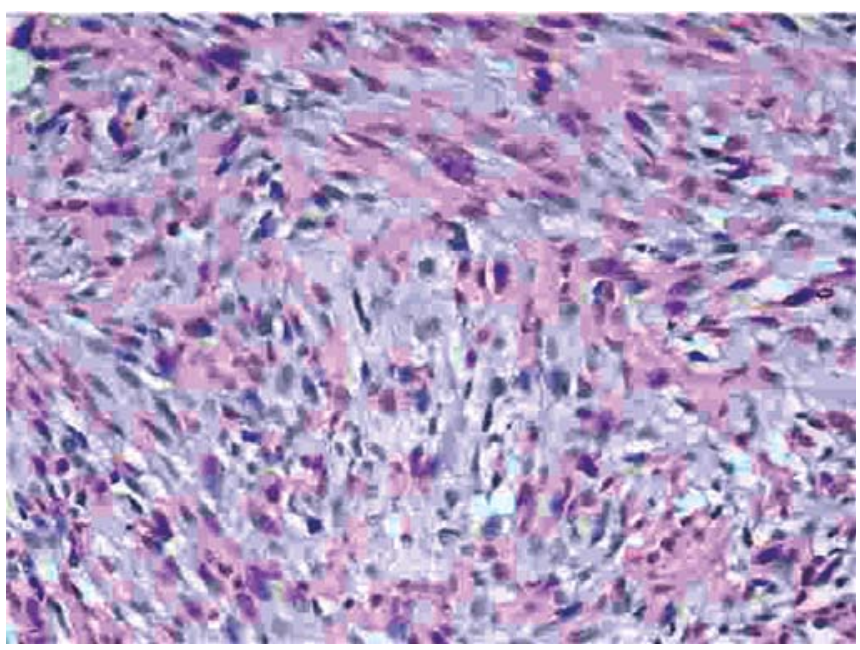

Figure 4: Photomicrograph of malignant fibrous histiocytoma showing Bizarre tumor cells with multinucleation (hematoxylin and eosin, X200) 
changes, and interlaced fibrosis. The tumor cells show oval to spindle shaped cells with moderate nuclear pleomorphism, vesicular nuclei with prominent nucleoli. Many interspersed bizarre multinucleate giant cells were also seen. Section from tissue sent as orbit showed an eyeball and surrounding soft tissue and optic nerve are free of tumor. IHC for CD34, HMB-45, desmin and S-100 is negative. Immunostain for cytokeratin and SMA were negative where as that for vimentin and CD68 were positive. All resection margins were free of tumor and orbit also was free of tumor.

Patient is on regular follow-up. He has total follow-up of 2 years and he last came for follow-up 2 months back. There is no evidence of locoregional recurrence.

\section{DISCUSSION}

Malignant fibrous histiocytoma (MFH) is a rare neoplasm of the maxilla. ${ }^{1}$ It was first reported in $1974 .^{3}$ Approx. 62 cases of malignant fibrous histiocytoma of maxilla have been reported in the international literature till date. Peak occurrence is in persons aged 50-70 years with slight male predominance. It can occur everywhere, owing to its mesenchymal origin. ${ }^{4}$ The most common sites of occurrence in the head and neck are the sinonasal tract, soft tissues of the neck, craniofacial bones, and salivary glands. ${ }^{5,6}$

Maxillary MFH exhibits broad range of histological patterns. Enzinger and Weiss have defined five histological subtypes of MFH as follows: (1) storiform-pleomorphic, (2) myxoid, (3) giant cell, (4) inflammatory, and (5) angiomatoid. ${ }^{7}$ The storiform-pleomorphic variant is the most common type. Tumors with angiomatoid and myxoid patterns are often associated with a more favorable prognosis as they metastasise late and respond well to surgery (Enzinger). The inflammatory and, the pleomorphic types are more aggressive, metastasise early and respond less favorably to surgery alone (Block). ${ }^{8}$ Our patient showed malignant tumor arranged predominantly in form of fascicular pattern and also in storiform pattern. Review of literature demonstrated that most cases were positive for vimentin and only few cases were positive for cytokeratin and desmin. ${ }^{9}$ In our case Immunohistiocytochemistry for CD34, HMB-45, desmin and S-100 was negative. Immunostain for cytokeratin and SMA was also negative whereas that for vimentin and CD68 was positive.

In recent times MFH is considered as a form of fibrosarcoma and the tumor is very likely over diagnosed by some pathologists. This is because the more pleomorphic the tumor, the more difficult is to distinguish from other types of sarcomas, such as pleomorphic leiomyosarcoma, pleomorphic liposarcoma and differentiated liposarcoma. Distinction among these pleomorphic soft tissue tumors is best achieved by a joint immunohistochemical and ultrastructural study.

Surgery is the best treatment for this aggressive neoplasm and radical neck dissection is an indicated when there is clinical evidence of lymph node metastasis. ${ }^{10}$ Most of the cases 54/61 (88.5\%) reported in literature review were treated surgically. Postoperatively both radiation therapy and chemotherapy have been used in few of the cases. Only radiotherapy or chemotherapy had poor results. Local recurrence rate of MFH after initial local excision ranges between $16 \%$ and $52 \% .{ }^{11}$ Block et al reported local recurrence or distant metastasis in $55 \%$ of cases of MFH. ${ }^{8}$ The presence of positive surgical margins after definitive treatment is the single most important factor relating to local recurrence. ${ }^{11}$ According to Barnes and Kanbour, $80 \%$ of patients with local recurrences after incomplete surgical treatment subsequently die from disease. ${ }^{12}$ Recurrence is related to size, depth of invasion, and microscopically positive surgical margins. From the review of literature we found 9/61 (14.7\%) recurrent cases and 3/61 (4.9\%) nonrecurrent cases while other cases, they did not mention about the recurrence. In 6/61 (9.8\%) cases of maxilla MFH reported metastasized distantly to lung, skin, bone, pleurae, pancreas, kidneys and bone marrows. ${ }^{13,14}$ In a review of oral soft tissue MFH, lung metastasis occurred in $44 \%$ of the patients. Only 6/61 (9.8\%) cases survived without disease while all other cases died with disease or lived with deteriorated condition. In our case the patient came with extensive local recurrence after 8 months of surgery and postoperative chemoradiotherapy. Extended radical maxillectomy with orbital exenteration was done followed by chemotherapy. Follow-up at 4 weeks interval showed no disease but follow-up at 2 months interval revealed local recurrence on nasal cavities bilaterally. Patient was advised revision surgery but lost to follow-up after that.

In conclusion, we believe that MFH of the maxilla may have poorer prognosis than those tumors in the other parts of the maxillofacial skeleton. Size, depth, histopathologic features, immunohistochemistry and ultrastructural features, surgical margin status and adjuvant radiotherapy or chemotherapy are the most important predictors of outcome.

\section{REFERENCES}

1. SW Wood, FM Enzinger. Malignant fibrous hystiocytoma tumor cells resemble fibroblasts. Am J Surg Pathol 1986;10:323-25.

2. KA Al-Salihi, KA AL-Jashamy, S Ab Rahman, AR Samsudin. Maxilla tuberosity malignant fibrous histiocytoma with giant fibroblastic cells. Oral oncology(extra) 2006;42:115-22.

3. GJ Spector, JH Ogura. Malignant fibrous hystiocytoma of maxilla. A case report of an unusual lesion. Arch Otolaryngol 1974;99:385-87. 
4. JEO Brien, AP Stout. Malignant fibrous xanthomas. Cancer 1963;17:1445-58.

5. K Watanbe, M Yambe, T Toh, M Ueda. Malignant fibrous histioma of maxilla. Oral Oncol Suppl 2005;1:203.

6. YW Chan, YC Guo, TL Tsai, CZ Lin. Malignant fibrous histiocytoma of maxillary sinus presenting as toothache. J Chin Med Assoc 2004;67:104-07.

7. SW Weiss, FM Enzinger. Malignant fibrous histiocytoma: An analysis of 200 cases. Cancer 1977;41:1672.

8. MS Block, JE Cade, FH Rodriguez Jr. Malignant fibrous histiocytoma in maxilla: A review of literature and report of case. Oral Maxillofacial Surg 1986;44:404-12.

9. JH Wang. Diagnosis and treatment of primary malignant fibrous histiocytoma of bone. Zonghua Wai Ke Za Zhi 1993;31:82-84.
10. SS sindhu, BP Bansal, H Prakash. Primary malignant fibrous histiocytoma of maxilla. J Dent 1978;6:261-64.

11. ME Pezzi, MS Rawling, JJ Esgro, et al. Prognostic factors in 227 patients with malignant fibrous histiocytoma. Cancer 1992;69:2098.

12. V Le doussal, JM Coindre, S Leroux. Prognostic factors for patients with localize primary malignant fibrous histiocytoma. A multicetric study of 216 patients with multivariate analysis. Cancer 1996;77:1823.

13. T Mentzel, E Galonji, C Wadden. Myofibrosarcoma. Clinicopathological analysis of 75 cases with emphasis on low grade variant. Am J Surg Pathol 1996;20:391-405.

14. FW Abdul-Karim, AG Ayala, SP Chawla, BS Jing, et al. Malignant fibrous histiocytoma of jaws. A clinicopathological study of 11 cases. Cancer 1985;56:1590-96. 